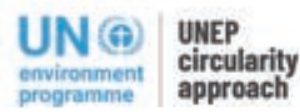

Procesos de la economia circular

Guia principal

Empresa-empresa

Usuario-empresa

Modelo de economia lineal producción

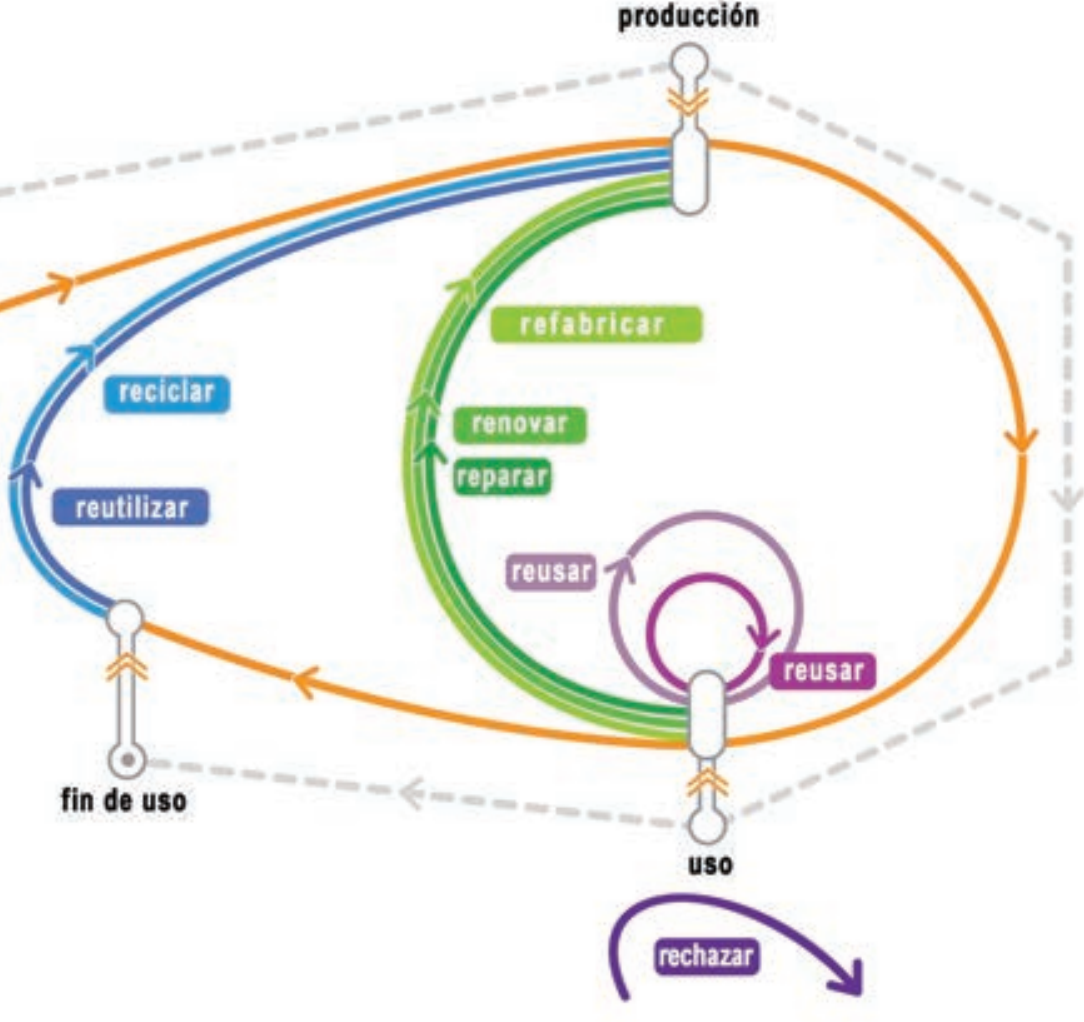

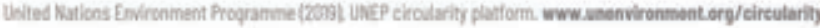




\section{Circularidad de los materiales: emulando a la naturaleza Manuel Quirós}

La transición de una economía lineal hacia un modelo circular trae consigo una serie de desafíos prácticos para toda la cadena de procesos: desde la extracción de la materia prima, su procesamiento, el diseño de productos o servicios, la fabricación, el uso y el fin de uso. Podríamos preguntarnos: ¿Cuáles son las estrategias para entender y aplicar este nuevo paradigma hacia una economía circular? Este artículo trata algunos aspectos, dentro de un marco de ideas en este complejo sistema circular, para avanzar y guiar de algún modo a diseñadores y emprendedores relacionados con los materiales en el cambio de paradigma. Se describen también algunas de las estrategias de la naturaleza desde una perspectiva sistémica y sus analogías potenciales de aplicabilidad.

\section{PALABRAS CLAVE}

Economía circular, diseño circular, biomímesis, bioinspiración, sostenibilidad, bioeconomía

\section{KEYWORDS}

Circular Economy, Circular Design, Biomimicry, Bioinspiration, Sustainability, Bioeconomy

El modelo actual económico-productivo que afecta a la totalidad del sistema global sea cual sea la actividad o el sector, continúa siendo mayoritariamente lineal. El proceso comienza con la extracción (destrucción) de recursos naturales vírgenes, que se convierten en materia prima que hay que transformar para ser convertidos en productos o servicios que serán empaquetados, distribuidos, vendidos y usados para tras su ciclo de uso, finalmente acabar en un vertedero. Este modelo globalmente aceptado durante décadas y actualmente cuestionado por numerosos aspectos como el crónico y colosal impacto ambiental y social que ocasiona durante todas sus fases hasta la incorporación realista de la finitud de los recursos, necesita ser reemplazado por otros donde se re-cuestione, nada menos que la totalidad del proceso. Las organiza-

\author{
Manuel Quirós \\ Master y Doctor en Biología, es Profesor de \\ Sostenibilidad y Biomimesis en la Escuela \\ de Diseño y Arquitectura del Instituto \\ de Empresa, así como Director del Slow \\ Fashion Lab. Colabora en numerosas \\ instituciones como la Universidad de \\ Navarra, la Cátedra de la Unesco de la \\ Universidad del País Vasco; la Universidad \\ de Maryland, entre otras. Es cofundador de \\ varios centros de estudio de la biomimesis \\ como son la Red Internacional de Estudios \\ e lnvestigación Biomimesis o de la \\ Biomimicry Iberia Association. Es editor \\ colaborador de la galardonada revista \\ Zygote Quarterly Journal. Conferenciante \\ requerido en foros internacionales como \\ la COP 25, la ONU-Habitat, escribe \\ habitualmente en un blog, único en \\ castellano, sobre la biomimesis. \\ E-Mail: info@natureinspireus.com
}

Fig. 01

Modelo circular según UNEP (2019). United Nations Environment Programme. 
Fig. 02

Modelo de economía circular según la Fundación Ellen McArthur. The Ellen MacArthur Foundation.

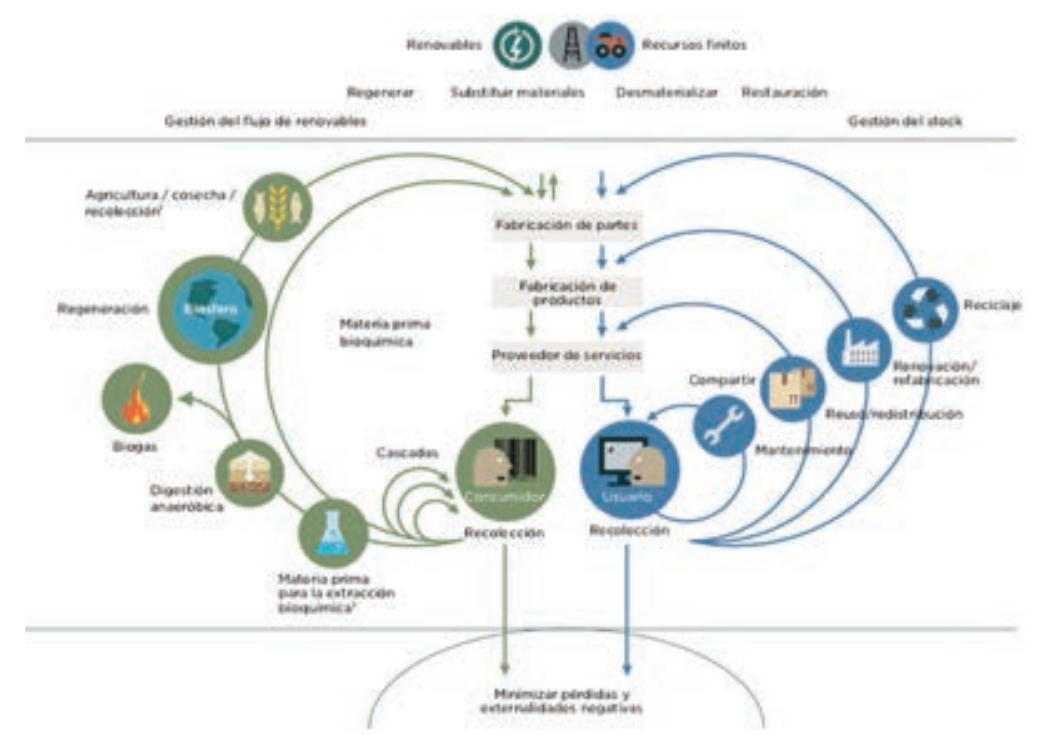

ciones gubernamentales, científicas o empresariales y financieras nos informan desde hace más de una década de una presión creciente sobre los recursos globales y el clima, debido a la principalmente a la actividad humana (IPCC, 2014; WBCSD, 2014; European Comission, 2018). La economía circular se considera un enfoque prometedor para ayudar a reducir la presión que ejerce la humanidad y que pretende encaminarse hacia una sostenibilidad global. Este modelo irrumpe con fuerza (European Comission, 2014) ya sea a través del diseño o la economía que acaba con el concepto de basura, incorporando cascadas donde los materiales ya sean biológicos o industriales se pueden reincorporar sin perder su valor y utilidad. Este modelo iniciado en los 80 por Börlin y Stahel (Börlin, 1987) y más adelante desarrollado por McDonough y Braungart (2002) y finalmente globalizado por la Fundación Ellen McArthur (2000), (fig. 02) y la propia O.N.U. (fig. 03) alcanza un grado mayor y profundo en torno a la sostenibilidad y regeneración que resulta muy interesante y esperanzador. El modelo además nos reconecta con la naturaleza, lo que somos, emulando el modo de operar de los sistemas naturales que llevan funcionando de modo sostenible y regenerativo desde hace 3.850 millones de años tal y como comprobamos por ejemplo en los ciclos del agua, carbono o el propio flujo de materiales, donde el concepto de basura no existe y es reemplazado por el de recursos (Quirós, 2016, 2019). La relación entre naturaleza y diseño es vital y debe aplicarse de modo consciente para mejorar la innovación y el camino hacia la sostenibilidad y la regeneración (Bar-Cohen, 2006). Este proceso se puede lograr considerando los principios del funcionamiento de los sistemas vivos entendiendo las estrategias de supervivencia en la naturaleza, la evolución y el crecimiento, así como a través de una comprensión más profunda y holística de las técnicas y procesos de supervivencia utilizados en los ecosistemas cada día. El modelo circular al que se hace referencia, se presenta como una oportunidad lógica de estrategias valiosas en el que se impulsan aspectos como el reciclaje, la reutilización sistemática, la prevención de pérdidas de materiales valiosos, el diseño ecológico, entendido como buen diseño, o la simbiosis industrial. Además podrán generar nuevos empleos, un crecimiento y desarrollo económico sos- 


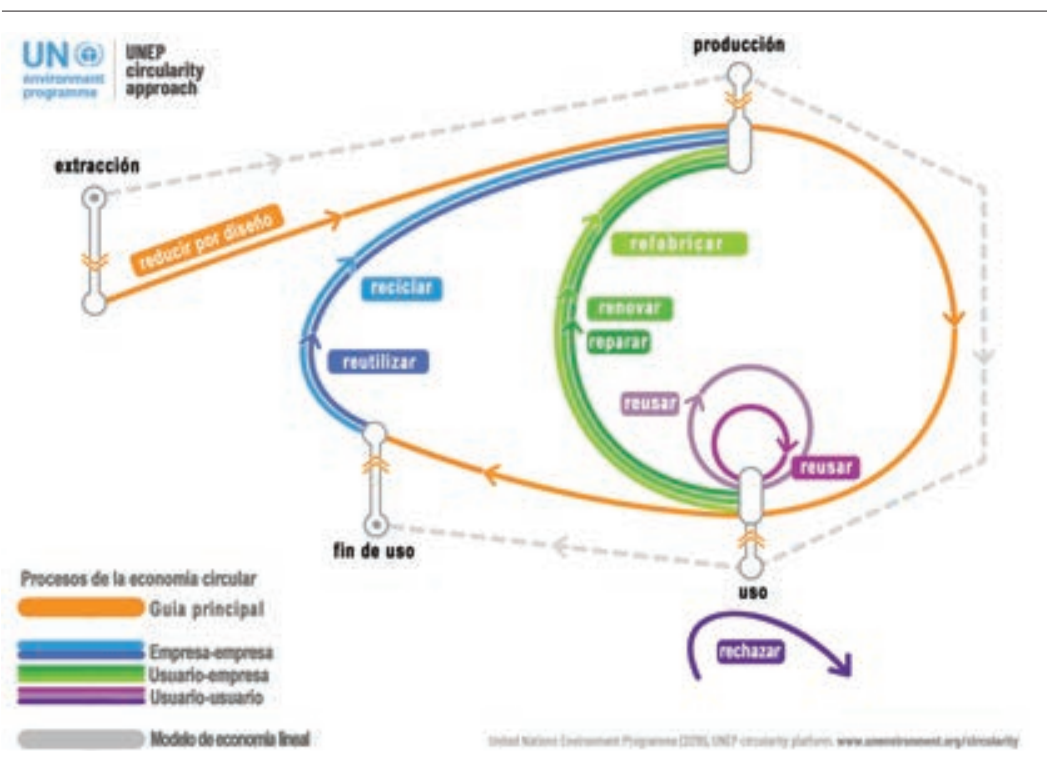

Fig. 03

Modelo circular según UNEP (2019).

United Nations Environment Programme.

tenido, mostrando nuevos modelos de negocio asociados, que pueden llevar a Europa, líder actual e impulsor, hacia una radical reducción de la basura que reduce el elevado exceso de emisiones de $\mathrm{CO}_{2}$ y $\mathrm{NH}_{4}$ y sus consecuencias climáticas, así como otros impactos ambientales. Este "nuevo" modelo en definitiva optimiza el sistema más allá del de los materiales, incluyendo un cuidadoso manejo de su flujo ya sea en el ciclo técnico donde son mantenidos lo máximo, reutilizados, refinados y en ultima instalación reciclados; o ya sea en el ciclo biológico, donde se potencia toda una cascada de materiales no tóxicos que podrían sin duda servir de abono a la productividad natural del suelo como concepto de capital natural. El ciclo biológico mencionado, hace referencia a materiales orgánicos empleados en los procesos industriales que tienen la capacidad intrínseca de retornar al ciclo natural regenerativo. Por otro lado, el ciclo técnico exige un potencial de desemsamblaje y reparabilidad en la ideación y diseño de productos que puedan tener el grado de recuperación en el flujo de materiales de cara al citado mantenimiento de la más alta calidad y durabilidad de los mismos.

En el diagrama mostrado en la figura 02 , los círculos más pequeños suponen los que más valor retienen y ha de ser entendido en 3 dimensiones como si de un cono se tratara visto desde arriba. El éxito del modelo, en clara expansión, implica el avance y entendimiento de sectores como el diseño industrial, el sector textil o la arquitectura, la logística inversa y sin duda la emergencia de nuevos modelos de negocio asociados. Se estima que puede tener un impacto en el ahorro de costes superior a los 600-700 billones de euros en Europa con independencia de la tipología de bienes y servicios de consumo (The Ellen MacArthur Foundation, 2012-2013) así como mitigar los riegos asociados a la incertidumbre en el precio dada la potencial volatilidad del suministro de materiales debido al cambio climático (Goddin y col., 2014; Informe Comisión Europea, 2014). Sectores como la electrónica, la automoción, los electrodomésticos o la industria textil, solo por citar algunos, son conocedores desde el año 2000 del aumento en el número de usuarios y la volatilidad asociada a la seguridad del suministro (The Mckinsey Commodity price index 1999-2001). 
Por otro lado, en el diseño circular para el ciclo tecnológico también es operativo en productos de servicio, de tal manera que los materiales o nutrientes técnicos pueden reciclarse de forma continua y segura en nuevos materiales o productos. Para establecer el flujo continuo de recursos en los denominados desperdicios o rechazos deben reciclarse en materiales que tenga propiedades equivalentes a las del material original. Esto requiere un reciclaje primario o uno terciario de materiales (McDonough, 2013) entendido estos como un reprocesamiento mecánico hacia otro producto con propiedades equivalentes. McDonough y Braungart hacen hincapié en que se debe mantener la calidad del material, y distinguen entre suprareciclaje e infrareciclaje en el que este último no permite un flujo cíclico de recursos, sino que solo retrasa el flujo lineal desde la producción hasta el desperdicio citado. Por lo tanto, el infrareciclaje implica que un material se reprocesa en un producto de bajo valor o de menor potencial valor. El suprareciclaje por el contrario, se refiere a retener o mejorar las propiedades del material, siendo este último concepto relativamente nuevo y poco explorado, aún con algunos interesantes casos en el sector textil como Elvis \& Kresse o Freitag, por citar algunos. En línea con esta distinción, los procesos que pueden etiquetarse como reciclaje cuaternario o reciclaje externo no se ajustarían a un enfoque circular para el diseño del producto según ciertos autores (Kumar y col., 2011).

El modelo circular no solo emula las estrategias naturales sino que construye un modelo basado en la eliminación del desperdicio/ basura y la contaminación asociada a los impactos negativos que ocasiona el negocio "como de costumbre" (b.a.u. business as usual por sus siglas en inglés). A esta nomenclatura hemos de añadir el consecuente impacto de los gases de efecto invernadero así como los que afectan a la vida terrestre, acuática y aérea.

Un segundo concepto de este modelo circular se fundamenta en el mantenimiento favorable de la calidad de los productos y sus materiales de cara a preservar su valor dentro del sistema (Steinmann, Z.J.N. y col. 2019). Finalmente, en el modelo circular subyace la regeneración de los sistemas naturales de los que dependemos, donde se fundamente el bienestar de los habitantes del planeta y la base de una economía global. Ya lo apuntó Ivon Chuinard, creador y dueño de la multinacional textil Patagonia: "no hay negocio posible en un planeta muerto" (Chuinard, 2016).

Sobre la base mencionada anteriormente iniciada por Stahel, McDonough y Braungart se sobrentienden 3 potenciales estrategias que no podemos subestimar: la ralentización de los bucles de recursos a través del diseño de bienes de larga duración, y ampliable a la extensión de la vida del producto en sí (por ejemplo a través de la reparación o la remanufactura/refabricación), donde el período de utilización de los productos se extiende y o intensifica, lo que resultaría un avance en una desaceleración del flujo de recursos necesarios; la potenciación de bucles cerrados de recursos que se retroalimentan evitando extracciones masivas de materiales vírgenes; un incremento de la eficiencia de los recursos entendida como un apoyo al punto anterior en la reducción en las fases iniciales del ciclo de vida de los productos, base actual de la maquinaria productiva.

La no acción y la consecuente ralentización en este aspecto hacia la circularidad, conlleva una serie de riesgos asociados que las empresas están ya incluyendo en sus estrategias y balances, ya 
que afectan a la cadena de valor implícita. Así, por ejemplo, los precios históricos de los materiales y/o de las proyecciones de precios futuros, suelen utilizarse para identificar el riesgo de la variación de precios y por tanto la perspectiva de volatilidad de los mismos (The McKinsey Global Institute; Duclos y col., 2010). Por otro lado, son bien estudiados desde hace tiempo los riesgos relacionados con la seguridad en la continuidad del suministro de un material concreto para un producto dado y que están relacionados con la disponibilidad de ese material para la compra por parte del fabricante del producto (IlbStudio, 2012, varias referencias). Pero en la realidad práctica se da una interacción compleja entre varios elementos como son la disponibilidad de un material, los mercados competidores para el uso de ese material, la oferta y demanda dentro de cada uno de esos mercados, las limitaciones regulatorias para la extracción legal, la estabilidad política de los estados ricos en un material dado y la capacidad de sus respectivos compradores de productos para absorber aumentos en el costo debido a estos múltiples factores. Procesos todos de una enorme complejidad a multiescala. De este modo el riesgo de la cadena de suministro puede estar asociado con una serie de factores externos como, por ejemplo, el monopolio de suministro, la existencia de imperativos legales, de gobiernos débiles, de ciertos estándares ambientales deficientes o fuentes de ciertos materiales como los minerales en conflicto (Unión Europea, 2014; Yale University, 2018).

Otro importante riesgo a tener en cuenta cada vez más considerado, es la escasez en la disponibilidad del suministro futuro y accesible en la corteza terrestre (UE 2010, Anexo V). Hay todo un debate sobre los conceptos de "escasez absoluta" y de "escasez económica" que implican que el coste de extracción de materiales se vuelve más costoso a medida que los recursos disponibles se consumen y el rendimiento típico disminuye como resultado del cambio climático y eventos ambientales severos e inesperados (huracanes, riadas, incendios, pandemias, ...). Finalmente mencionar el creciente riesgo asociado a la toxicidad del material, cada día más sujeto a la normativa vigente y muy susceptibles a futuras restricciones y que, por tanto, también puede interrumpir el uso prolongado de ciertos materiales, limitando su uso potencial así como el valor económico futuro. En la Unión Europea (UE en adelante), el vigente Reglamento REACH (REACH, 2006 de sus siglas Reglamento sobre Registro, Evaluación, Autorización y restricción de productos Químicos) también promueve métodos alternativos para la evaluación de riesgos de sustancias potencialmente peligrosas y dañinas. En este aspecto añadir la Directiva RoHS (RoHS, UE 2011) también de la UE que prohíbe la colocación en el mercado de nuevos equipos eléctricos y electrónicos que contengan más de los niveles acordados de plomo, cadmio, mercurio, cromo hexavalente, polibromados, retardantes de llama con bifenilos (PBB) y difenil éteres polibromados (PBDE). Otro marco de referencia es el programa iSustitúyalo ahora! (SIN List, 2014) de la Lista de la Secretaría Internacional de Química, basada en los criterios establecidos por la regulación de productos químicos de la UE que ha identificado ciertas sustancias de muy alta preocupación. Finalmente la lista de productos químicos prohibidos Cradle to Cradle Certified ${ }^{\mathrm{TM}}$ (C2C, 2013) hace referencia a sustancias que contengan más de 1000 ppm debido a su tendencia a acumularse en la biosfera y conducir a efectos negativos irreversibles para la salud de los seres vivos incluyendo la especie humana. 
Quisiera incluir brevemente un aspecto poco conocido aún por la industria y los profesionales del diseño, que es la biomímesis. Una disciplina disruptiva de diseño y de gestión estratégica para las empresas, sea cual sea el sector, en el que se emulan los funcionamientos de los organismos vivos a diferentes escalas (órgano-especie-ecosistema) y niveles (micro-macro-meso) para encontrar y ampliar el campo de las soluciones a los múltiples retos a los que nos enfrentamos en este siglo. Por cuestión de espacio solo mencionaré lo que es conocido entre los profesionales y seguidores de la disciplina biomimética como los Principios de Vida (PV en adelante), (fig. 04); (Baumeister y col., 2012), que otras escuelas europeas han denominado Principios de diseño inspirado en la Naturaleza (Tempelman y col., 2015). Los PV representan un patrón encontrado en multitud de especies vivas que nos proporcionan estrategias innovadoras, inspiradoras y creativas para lograr sobrevivir, creando un modelo diferente al actual vigente. Ajustarnos a esas leyes resulta todo un reto de obligado cumplimiento e innegociable, no solo para nosotros, sino también sobre las maneras de cómo podemos contribuir a la propia salud del sistema terrestre del que dependemos (Quirós, 2013). De este modo vemos las interconexiones, el vínculo, entre las especies y sus hábitats. La naturaleza así nos muestra la medida de los límites que no debíamos haber sobrepasado, pues por primera vez en la historia de la humanidad nos hemos convertido en "hacedores" del clima, siendo capaces de alterarlo a escala global y sistémica (Rockstrom y col., 2009). En el centro del diagrama (fig. 04) leemos, "la vida crea condiciones que conducen a la vida" que representa un mensaje fundamental, un mantra, que aplica en cualquier acción que nuestra disciplina laborar desarrolle. Podremos así preguntarnos: ¿“mi negocio/diseño/propuesta crea condiciones favorables para la vida?. Los PV pueden inspirarnos para encontrar respuestas en el modelo natural. A través de 6 principios subdivididos en una serie de subprincipios que nos guían de un modo estratégico para entender y aplicar con el objetivo de crear soluciones sostenibles y regenerativas tal y como lo lleva haciendo la vida en la Tierra desde hace millones de años. En la capa externa leemos una serie de leyes que inexorablemente operan en el sistema terrestre y que no pueden ser ignorados. Solamente si consideramos las lecciones de la naturaleza en un contexto sistémico, podremos asegurar que nuestros diseños serán adecuados para el objetivo que perseguimos del desarrollo sostenible. Este es un paso clave en la práctica de la biomímesis y lo que la distingue de una categoría más amplia de diseño bioinspirado. La aplicación de estos principios requieren tiempo, pero una vez que se comienza a entender estos patrones, se debe empezar a aplicar al inicio de cualquier proceso de diseño, en la fase de establecer el alcance, y seguir ponderándolos y usándolos a lo largo de todo el resto de las fases de ideación y desarrollo. La meta es traducir estas lecciones naturales en especificaciones de diseño, métricas de control de calidad, selección de materiales y otras opciones de manufactura o de proceso. Aunque con frecuencia implica cierto grado de complejidad y la aplicación total de los 6 PV presenta limitaciones vigentes, pero reproducir todos estos patrones es una excelente meta aspiracional para cambiar el modo en el que operamos actualmente. Es interesante entender la multidisciplinariedad en la aplicación de esta disciplina en los que se ha de incorporar en la mesa de decisiones a los biólogos junto con diseñadores, ingenieros, economistas, etc. 


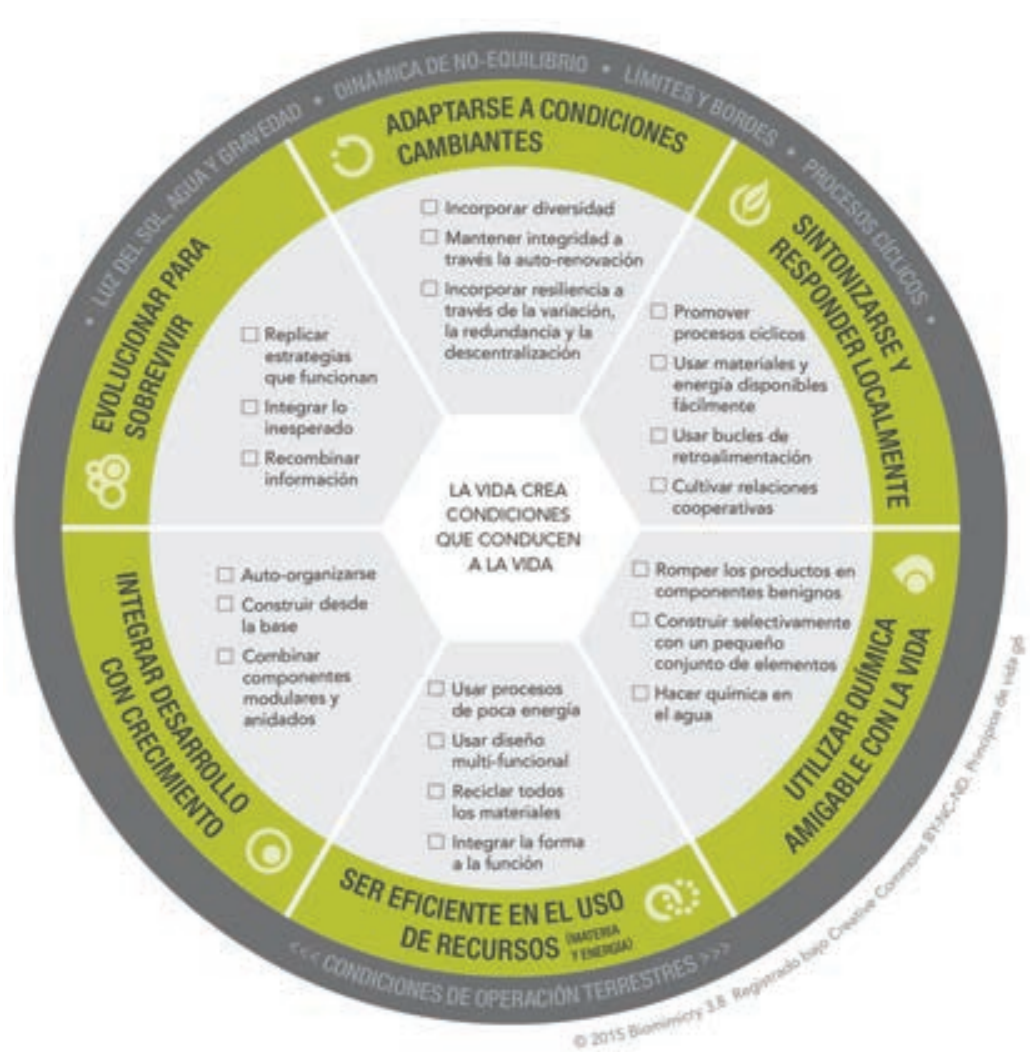

Fig. 04

Principios de Vida o estrategias de supervivencia de la naturaleza.

Biomimicry Institute ${ }^{\circledR}$. Principios de Vida. Biomimicry 3.8.

El incesante aumento de la demanda de materiales y productos va a suponer un cuello de botella a medida que la clase media se hace más accesible a nivel económico (Kharas y Hamel, 2018). La mayor parte de este material está hecho de recursos finitos que se emplean una sola vez y directamente pasan a ser basura. Un claro ejemplo de esta tipología de material/producto es el que se emplea masivamente en el embalaje de productos con poliestireno para proteger el envío y entregas de equipos frágiles y no tan frágiles. El poliestireno está hecho de petróleo y se usa principalmente una sola vez. Es voluminoso y se degrada en cientos de años por la acción de la luz, el agua y el viento ocasionando enormes problemas en este período a corto, medio y lago plazo. Técnicamente es reciclable, pero bajo un elevado coste económico y ambiental y su reciclaje no es económicamente viable. Paralelamente a esta realidad, y aplicando la emergencia ambiental hacia la circularidad, tenemos acceso a grandes cantidades de subproductos agrícolas que son descartados o infrautilizados y que son fuente de aplicabilidad potencial de la circularidad descrita en el presente artículo. Bayer y McIntyre (ecovativedesign.com), fundadores de Ecovative Design, encontraron inspiración mientras observaban los hongos que crecían en las astillas de la madera y cómo el micelio funcionaba. El micelio es la parte vegetativa de los hongos, formado por cientos de microfibras entrelazadas producidas por las esporas, y que lo convierte en un material increíblemente fuerte cuando se seca, siendo un análogo en su funcionamiento a un pegamento natural y auto-ensamblable. Esto permitió al equipo formular un nuevo método para producir materiales capaces de reemplazar varios tipos de productos, incluidos plásticos expandidos a base de petróleo y tableros de partículas fabricados con formaldehídos, 
Fig. 05

Esquema de proceso de fabricación comparado entre un material convencional y otro circular. Modificado de Ecovative ${ }^{\circledR}$. Ecovative Design.

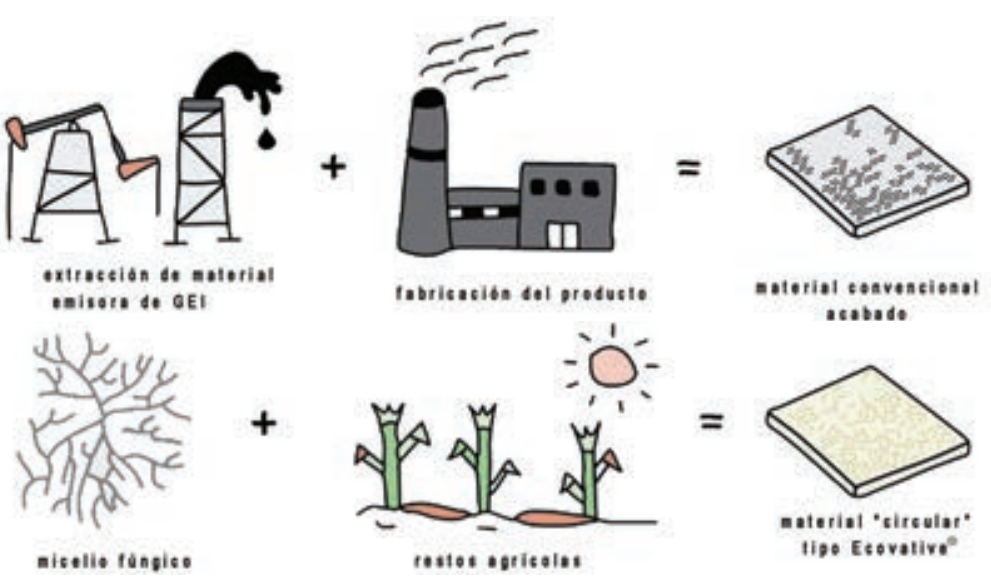

conocidos por su capacidad cancerígena. Desde 2007, Ecovative, una empresa con sede en Nueva York, ha estado sembrando residuos agrícolas con el micelio, de los hongos, colocando este material en diferentes moldes en función del producto deseado (fig. 05). El micelio si se combina con los desechos agrícolas en los moldes, el cultivo resultante puede formar los objetos que se deseen desde ladrillos, protectores de material delicado para transporte, mobiliario, objetos decorativos e incluso material ignífugo para múltiples usos. Al ser completamente orgánico en su composición, el hongo-material vegetal al final del ciclo de uso, puede fácilmente ser retransformado en otro nuevo o en su descarte para volver al ciclo del carbono. El micelio fúngico, alimentado por nutrientes procedente de estos desechos, extiende su red a través de la materia orgánica disponible, uniéndola firmemente y tras varios días de crecimiento, este es detenido por el efecto de un choque térmico produciendo las formas deseadas sin tener efecto alguno para la salud de los usuarios (Karana y col., 2018; Ikea; Ecovative).

De este modo se pueden ya sustituir los materiales tóxicos y de difícil reciclabilidad como son los poliestirenos expandidos (EPS), polipropipeno expandido (EPP) o el polietileno expandido (EPE) de amplísima aplicación aún hoy día. Señalar que el micelio crece en 5-7 días sin necesidad de luz o agua, digiriendo el subproducto agrícola pasando por un sencillo proceso de deshidratación y tratamiento térmico para detener el crecimiento y garantizar la ausencia de esporas o alérgenos.

Dentro de esta categoría de procesos ya podemos incluir a medida de que el sector industrial de manufactura se implique, gran variedad de recursos naturales no empleados hasta ahora como son microorganismos vivos, como es el caso citado que incluiría otros hongos o bacterias; materiales a base de plantas o árboles en forma de fibras vegetales varias, desechos del sector agrario, algas y mezclas derivadas de plantas, animales y microorganismos solo por citar algunos. Empresas como Dell, Steelcase, H\&M o lkea, están ya ampliando las miras con la garantía que puede proporcionar por ejemplo la certificación Cradle to Cradle. Mientras tanto Ecovative ya se mueve a sectores del aislamiento para la construcción, textiles (Mycoflex ${ }^{\mathrm{TM}}$ ), envases y alimentación.

Lo que comenzó siendo un laboratorio de ideas hoy se encamina a una realidad empresarial de enorme potencial y desarrollo. Su expansión está asegurada y la creatividad para la conexión entre empresa y naturaleza está a la espera. RA 


\section{Referencias bibliográficas}

- BAR-COHEN, Y., Biomimetics: using nature to inspire human innovation. Bioinspiration Biomimetics 1(2006) pp. 1-12.

-BAUMEISTER, D., Tocke, R., Dwyer, J., Ritter, S y Benyus, J. . Biomimicry Resource Handbook: A Seed Bank of Best Practices. Missoula, Biomimicry 3.8, 2012, p. 326.

-BÖRLIN, M. \& STAHEL, W.R., Economic Strategic for Durability. Swiss Bank Corpòration, Basel, Bankverein-Heft Nr. 32, 1987, p. 77.

- Cradle to Cradle Products Innovation Institute, Cradle to Cradle Certified ${ }^{\text {TM Banned List of }}$ Chemicals, 2013 (see http://www.c2ccertified. org/resources/detail/cradle-to-cradle-certified-banned-list-ofchemicals/).

- ChemSec, SIN (Substitute It Now!) List, 2014, http://sinlist.chemsec.org/.

- CHUINARD, I. 2016. Let my people go surfing. Edit.Penguin. p. 272.

- Directive on the restriction of the use of certain hazardous substances in electrical and electronic equipment, Dir. 2011/65/EU.

- Ecovative: [https://ecovativedesign.com/]. Último acceso el 18 de febrero de 2020.

-European Commission, Report of the Adhoc Working Group on defining critical raw materials, 2014.

- European Commission, 2014; Annex V to the Report of the Ad-hoc Working Group on defining critical raw materials, 2010 (available at http://ec.europa.eu/enterprise/policies/rawmaterials/files/docs/report-b_en.pdf).

- European Commission, Moving towards a circular econ-omy. Available online at: http:// ec.europa.eu/environment/circular-economy/ (accessed October 2014).

- Financing a Sustainable European Economy. Final Report 2018 by the High-Level Expert Group on Sustainable Finance. European Comission.

- GODDIN, J.R., MARTIN, W., Marshall, K. y Clifton,A. Identifying Supply Chain Risks for Critical and Strategic Materials, Shechtman International Symposium, 2014.

- IlbStudio, 2012. [ https://www.americanscientist.org/article/circular-visualizations]. Último acceso el 4 de febrero 2020.

- Ikea [https://www.intelligentliving.co/ ikea-mushroom-based-packaging/]. Último acceso el 18 de febrero de 2020.

-IPCC, Summary for policymakers, in O. R. EDENHOFER, Y., PICHS-MADRUGA, E. SOKONA, S., FARAHANI, K. KADNER, A. SEY-
BOTH, I., ADLER, S., BAUM, P., BRUNNER and B. EICKEMEIER (eds), Climate Change 2014, Mitigation of Climate Change. Contribution of Working Group III tothe Fifth Assessment Report of the Intergovernmental Panel on Climate Change, Geneva, 1-18, 2014.

- KARANA, D., BLAUWHOFF, D., HULTINK, E. J., SERENA CAMERE, "When the Materia Grows: A Case Study on Designing (with) Mycelium-based Materials". International Journal of Design 12(2):119-136. 2018.

- KHARAS, H. y HAMEL, K. A global tiping point: Half the world is now middle class or wealthier. 2018. Brookings Institution. [https://www.brookings.edu/ blog/future-development/2018/09/27/aglobal-tipping-point-half-theworld-isnow-middle-class-or-wealthier/?utm campaign=Brookings\%2Brief\&utm_sourc $\mathrm{e}=$ hsemail\&utmmedium =email\&utm_content $=66298094]$. Último acceso el 4 de abril 2020. Kumar, S., A. K. Panda and R. K. Singh, "A review ontertiary recycling of high-density polyethylene to fuel", Resources, Conservation and Recycling, 55, 893-910 (2011).

\section{- MCDONOUGH, W. and M. BRAUNGART,} The Upcycle: Beyond Sustainability-Designing for Abundance, North PointPress, NY, (2013), p. 227.

- MCDONOUGH, W. and M. BRAUNGART, Cradle to Cradle: Remaking the Way We Make Things, North Point Press, NY (2002).

- KUMAR, S., A. K. PANDA and R. K. SINGH, "A review on tertiary recycling of high-density polyethylene to fuel", Resources, Conservation and Recycling, 55, 893-910 (2011).

- QUIRÓS, M., "Biomimesis para la innovación social y la regeneración ambiental. Re-descubriendo el mundo natural. La Biomimesis en perspectiva”. Publicado por la Universidad Nacional Abierta y a Distancia (UNAD) de Colombia. p. 428, 2019. ISBN: 978-958-651641-9 || e-ISBN: 978-958- 651-631-0 19.

- QUIRÓS, M., (2016). "Biomimesis en el diseño: más allá de la sostenibilidad en el siglo XXI". En: Biomímesis: inspiración creativa en la naturaleza y escenarios potenciales de sostenibilidad. Memorias del Simposio internacional de estudios biomiméticos (Leticia-Amazonas, 27-29 de abril de 2016). ISSN 2590-8502. ISBN 978-84-617-7390-9.

- QUIRÓS, M., Los principios de Vida. 2013. En [https://natureinspireus.wordpress. com/2013/11/21/los-principios-de-la-vida/]. Último acceso el 11 de marzo de 2020.

- ROCKSTROM, J., W. STEFFEN, K. NOONE, A. PERSSON, F. S. CHAPIN, III, E. LAMBIN,

T. M. LENTON, M. SCHEFFER, C. FOLKE, H.
SCHELLNHUBER, B.NYKVIST, C. A. DE WIT, T. HUGHES, S. VAN DER LEEUW, H. RODHE, S. SORLIN, P. K. SNYDER, R. COSTANZA, U. SVEDIN, M. FALKENMARK, L.KARLBERG, R. W. CORELL, V. J. FABRY, J. HANSEN, B. WALKER, D. LIVERMAN, K. RICHARDSON, P. CRUTZEN, AND J. FOLEY. (2009). "Planetary boundaries: exploring the safe operating space for humanity". Ecology and Society 14(2), 1-32.

- REACH Legislation (see http://echa.europa. eu/regulations/reach/legislation), Regulation, Registration, Evaluation, Authorisation and Restriction of Chemicals, EC No 1907/2006, in particular Article 33

- TEMPELMAN, E., DER GRINTEN, B. VAN, MUL, E-J. Y PAUW. I. Nature Inspired Design. 2015. TU Delft. p. 145.

- The McKinsey Global Institute publishes historic price data, variation and volatility statistics for a number of commodities at http://www.mckinsey.com/insights/energy_resources_materials/resource_revolution_tracking_global_commodity_markets/.

- The Ellen MacArthur Foundation, Towards the Circular Economy, Volume1y 2, 2012-2013.

- DUCLOS S.J., OTTO J. P. and KONITZER D. G., Design in an Era of Constrained Resources, Mechanical Engineering-CIME, Sept. 2010.

-STEINMANN, Z.J.N, HUIJBREGTSA, M.A.J. AND REIJNDERSBA, L., "How to define the quality of materials in a circular economy?" Resources, Conservation \& Recycling 141 (2019) 362-363.

- Yale University, Environmental Performance Index, 2018. [ http://epi.yale.edu]. Último acceso el 26 de febrero de 2020.

-World Business Council for Sustainable Development (WBCSD). Vision 2050: The new agenda for business.Avalable online at: http://www. wbcsd.org/pages/edocu ment/edocumentdetails.aspx?id=219\&nosearchcontextkey= true (accessed December 20 World Business Council for Sustainable Development (WBCSD). Vision 2050: The new agenda for business. Available online at: http://www.wbcsd.org/pages/edocu ment/edocumentdetails. aspx?id=219\&nosearchcontextkey= true (accessed December 20 World Business Council for Sustainable Development(WBCSD). Vision 2050: The new agenda for business.Available online at: http://www.wbcsd.org/pages/edocument/edocumentdetails.aspx?id=219\&nos earchcontextkey=true (accessed December 2014).
RA. Revista de Arquitectura Núm. 22 - 2020

P. 46-55 